\title{
Switching of A Sixteen Electrode Array for Wireless EIT System Using A RF-Based 8-Bit Digital Data Transmission Technique
}

\author{
Tushar Kanti Bera, J. Nagaraju \\ Department of Instrumentation and Applied Physics, Indian Institute of Science Bangalore \\ Bangalore - 560012, Karnataka, INDIA, \\ Email: solarjnr@isu.iisc.ernet.in
}

\begin{abstract}
Surface electrode switching of 16-electrode wireless EIT is studied using a Radio Frequency (RF) based digital data transmission technique operating with 8 channel encoder/decoder ICs. An electrode switching module is developed the analog multiplexers and switched with 8-bit parallel digital data transferred by transmitter/receiver module developed with radio frequency technology. 8-bit parallel digital data collected from the receiver module are converted to 16-bit digital data by using binary adder circuits and then used for switching the electrodes in opposite current injection protocol. 8-bit parallel digital data are generated using NI USB 6251 DAQ card in LabVIEW software and sent to the transmission module which transmits the digital data bits to the receiver end. Receiver module supplies the parallel digital bits to the binary adder circuits and adder circuit outputs are fed to the multiplexers of the electrode switching module for surface electrode switching. $1 \mathrm{~mA}, 50 \mathrm{kHz}$ sinusoidal constant current is injected at the phantom boundary using opposite current injection protocol. The boundary potentials developed at the voltage electrodes are measured and studied to assess the wireless data transmission.
\end{abstract}

Keywords: electrical impedance tomography, EIT, wireless-EIT, Radio Frequency (RF), surface electrode switching, opposite current injection protocol, digital data transmission, phantoms, boundary potential data.

\section{Introduction}

Electrical Impedance Tomography (EIT) [1-3] is an image reconstruction technique in which the electrical conductivity or resistivity of a conducting domain is reconstructed from the surface potential developed by a current signal injected at the domain boundary. EIT finds good applications in different areas of science and technology due to its several advantages [1-3] over other computed tomographic techniques [4]. As a nondestructive testing modality, electrical impedance tomography has been extensively researched in clinical diagnosis [5-8], biomedical engineering [9] and biotechnology [10]. Wireless data acquisition (Fig.-1) has many advantages and hence it is sometimes preferred in medical EIT systems for electric isolation property and maximum patient safety. Surface electrodes are required to be switched in a particular fashion depending on the current injection protocol used in EIT. Analog multiplexers are used for electrode switching in modern digital EIT hardware [11]. Parallel digital bits are required to operate the multiplexers for electrode switching. More the electrodes in an EIT system, more digital bits are required to operate the suitable multiplexers. In wireless EIT, required parallel digital data are transmitted wirelessly from the computer end and it is received at the phantom side. Hence, more the digital bits required to operate the suitable multiplexers in EIT, more number of channels are required in encoder/decoder ICs.

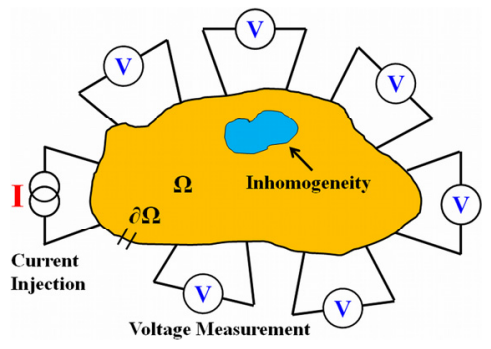

Fig. 1. Current injection and voltage measurement in EIT.

In this context a Radio Frequency (RF) transmission technology [12] based digital data transmission technique operating with 8 channel encoder/decoder is proposed to switch the surface electrodes of a 16-electrode wireless EIT system. 8-bit parallel digital data are generated and transmitted through the wireless transmission module (Fig.-2) and then the 8-bit data are converted to the 16-bit parallel digital data required for the multiplexer operation in the 16-electrode EIT system. 8-bit parallel digital data collected from the receiver module are converted to 16-bit digital data by using binary adder circuits and then used for switching the surface electrodes in opposite current injection protocol. 


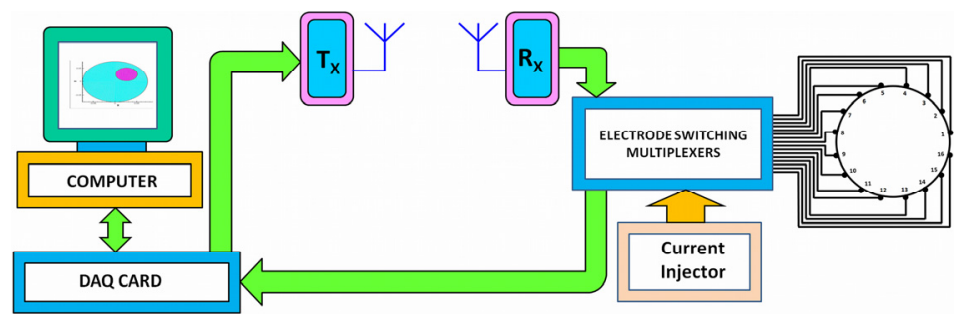

Fig. 2. A wireless digital data transmission scheme developed with Radio Frequency (RF) based transmitter (Tx) and receiver (Rx) modules for a 16-electrode EIT.

8-bit parallel digital data are generated using NI USB 6251 card in LabVIEW platform and sent to transmission module which transmits the 8-bit digital data digital data to the receiver side. Receiver module supplies the 8-bit parallel digital data bits to the binary adder circuits. Binary adder circuits convert the 8-bit parallel digital data to the 16-bit digital data and fed to the four multiplexers in electrode switching module. $1 \mathrm{~mA}, 50 \mathrm{kHz}$ sinusoidal current is injected at the phantom boundary using opposite current injection protocol and the boundary potentials developed at the voltage electrodes are measured and studied to assess the performance of the wireless data transmission module.

\section{Materials and Methods}

\subsection{Analog Instrumentation}

An analog EIT instrumentation [13] consists of constant current injector (Fig.-3), electrode switching module, signal conditioners and data acquisition system is used for boundary data collection. Constant current injector consists of a Voltage Controlled Oscillator (VCO) and a Voltage Control Current Source (VCCS) as shown in Fig.-3.

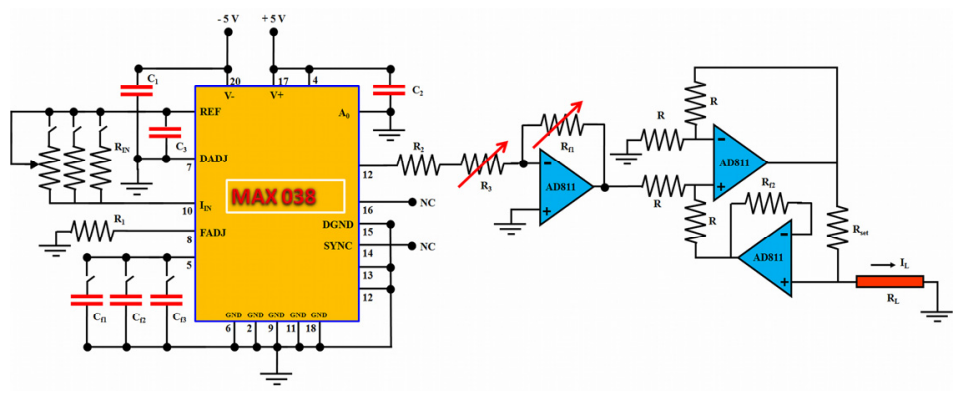

Fig. 3. Schematic of the constant current injector developed with a voltage controlled oscillator and modified Howland constant current source.

The VCO consists of a variable frequency function generator IC (MAX038, Maxim Inc. USA) and supplies the input signal to the VCCS. MAX038 is a high-frequency, precision function generator producing accurate, highfrequency triangle, sawtooth, sine, square, and pulse waveforms with a minimum of external components [14]. VCO generates signal with different frequency bands depending on the value of $\mathrm{C}_{\mathrm{F}}$. Frequency of the VCO output is a function of $\mathrm{R}_{\mathrm{IN}}$ and $\mathrm{C}_{\mathrm{F}}$ with a positive dc supply of \pm 5 volts [13]. Different capacitors are connected and individually switched for different frequency bands. For a particular frequency, one capacitor is connected as $C_{F}$ using single pole single throw DIP switches along with an adjusted value of $R_{I N}$. Changing the value of the $\mathrm{R}_{\mathrm{IN}}$ and $\mathrm{C}_{\mathrm{F}}$ in the VCO circuit a sinusoidal voltage signal of a very high bandwidth $(10 \mathrm{~Hz}-10 \mathrm{MHz})$ is achieved [13] which is suitable for a multi frequency EIT application. VCCS is basically a modified Howland constant current generator (Fig.-3) which is fed by the VCO. VCCS is developed with two AD811 ICs (Analog Devices Inc. USA) [15]. AD811 is a high speed wideband current feedback operational amplifier which is suitably used in modified Howland current source with a feedback resistor of $750 \Omega$ [15] in voltage follower circuit of the VCCS (Fig.-3). A signal conditioner block consisting a $50 \mathrm{~Hz}$ notch filter and a narrow band pass filter with a center frequency of $50 \mathrm{kHz}$ is used for improving the signal to noise ratio of the measured potential. 
An electrode switching module is developed with high speed CMOS analog multiplexers (MUX) (CD4067BE). Four 16:1 MUXs are used for current electrode and voltage electrode switching with their similar pins (Pin-1 through Pin-16) shorted and connected to the corresponding electrodes on the phantom. The electrode switching module is developed with four CD4067 ICs; two for current electrode switching and another two for voltage electrode switching. 4-bit parallel digital data are used to operate one multiplexer and hence, 16-bit parallel digital data are used to operate four 16:1 MUXs. Current electrodes are switched with two multiplexer named as current electrode multiplexers (MUX-I $I_{1}$ and $\left.M U X-I_{2}\right)$. Voltage electrodes are switched with two multiplexer named as voltage electrode multiplexers (MUX-I $\mathrm{I}_{1}$ and $\left.\mathrm{MUX}-\mathrm{I}_{2}\right)$. Positive terminal of the current source is connected to the input terminal of MUX-I $I_{1}$ where as the other point of the VCCS is connected to the input terminal of MUX-I ${ }_{2}$. Similarly, the positive terminal of the voltage measuring probe is connected to the input terminal of MUX- $\mathrm{V}_{1}$ where as the other point of the voltage measuring probe is connected to the input terminal of MUX-V.

\subsection{RF Transmitter/Receiver Module}

A wireless RF transmission system (Fig.-4a) is developed using ASK (Amplitude Shift Keying) based transmitter/receiver $\left(\mathrm{T}_{\mathrm{x}} / \mathrm{R}_{\mathrm{x}}\right)$ pair operating at $433 \mathrm{MHz}$. 8-bit parallel digital data are generated in DAQ card and fed it to the encoder IC (HT640) in the transmitter module. Encoder converts the parallel data into serial format and then feeds it to the transmitter block which transmits the serial data to the receiver end (Fig.-4a). Receiver receives the serial data and supplies it to the decoder IC (HT648L) which converts the serial data into parallel data again.

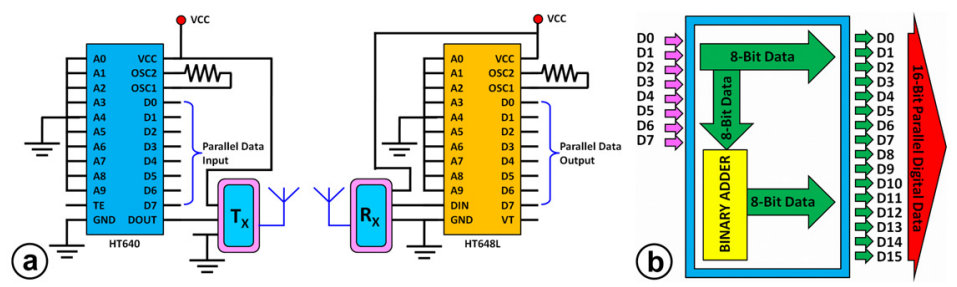

Fig. 4. (a) RF transmitter (Tx)/receiver (Rx) module, (b) Schematic of the binary adder circuit.

\subsection{Binary Adder Circuit}

8-bit parallel digital data received from the wireless transmission module are converted to the 16-bit digital data using the binary adder circuits (Fig.- $4 b)$. 8-bit parallel digital data $\left(\mathrm{D}_{7} \mathrm{D}_{6} \mathrm{D}_{5} \mathrm{D}_{4} \mathrm{D}_{3} \mathrm{D}_{2} \mathrm{D}_{1} \mathrm{D}_{0}\right)$ collected from the receiver module and fed to the binary adder circuits. In binary adder circuit the 8-bit parallel digital data are converted into 16-bit digital data $\left(\mathrm{D}_{15} \mathrm{D}_{14} \mathrm{D}_{13} \mathrm{D}_{12} \mathrm{D}_{11} \mathrm{D}_{10} \mathrm{D}_{9} \mathrm{D}_{8} \mathrm{D}_{7} \mathrm{D}_{6} \mathrm{D}_{5} \mathrm{D}_{4} \mathrm{D}_{3} \mathrm{D}_{2} \mathrm{D}_{1} \mathrm{D}_{0}\right)$ required for the multiplexer operation. 8-bit parallel digital data are summed with another 8-bit digital data $\left(\mathrm{S}_{7} \mathrm{~S}_{6} \mathrm{~S}_{5} \mathrm{~S}_{4} \mathrm{~S}_{3} \mathrm{~S}_{2} \mathrm{~S}_{1} \mathrm{~S}_{0}\right)$. $\mathrm{S}_{7} \mathrm{~S}_{6} \mathrm{~S}_{5} \mathrm{~S}_{4} \mathrm{~S}_{3} \mathrm{~S}_{2} \mathrm{~S}_{1} \mathrm{~S}_{0}$ data are defined by the type of current injection protocol used in the EIT system. Binary adder outputs (16-bit parallel digital data) are fed to the multiplexer (MUX-I $I_{1}$ MUX-I ${ }_{2}, M U X-V_{1}$ and $M U X-V_{2}$ ) used for switching the surface electrodes in opposite current injection protocol.

\subsection{Data Acquisition System}

The data acquisition system consists of a NI USB-6251 DAQ card which collects the boundary data through the multiplexer board connected to the surface electrodes. Data acquisition software is written in LabVIEW and interfaced with the DAQ card through PC. 8-bit parallel digital data are generated using NI USB 6251 card in LabVIEW platform and sent to transmission module which transmits the 8-bit digital data digital data to the receiver side. Receiver module supplies the 8-bit parallel digital data bits to the binary adder circuits. Binary adder circuits convert the 8-bit parallel digital data to the 16-bit digital data and fed to the four multiplexers in electrode switching module.

\subsection{EIT-Phantom}

A reconfigurable practical phantom (Fig.-5a) is developed with a shallow glass tank (150 mm diameter) and sixteen stainless steel electrodes [16] equally spaced on the tank inner wall (Fig.-5a) and fixed on tank wall using steel paper clips. Sixteen identical electrodes (rectangular shape, $34 \mathrm{~mm} \times 10 \mathrm{~mm}$ ) are cut from a $50 \mu \mathrm{m}$ thick high quality stainless steel (type 304) sheet. All the electrodes are connected to the EIT electronic hardware through the low resistivity flexible multi-strand copper wires with steel crocodile clips (Fig.- 5a). 

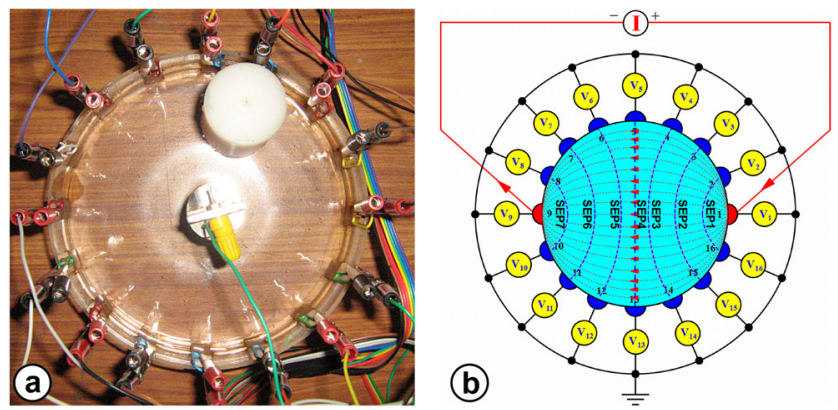

Fig. 5. (a) Practical phantom with nylon inhomogeneity near electrode no. 4, (b) Boundary potential measurement for opposite current injection.

The phantom tank is filled with a $0.9 \%(\mathrm{w} / \mathrm{v}) \mathrm{NaCl}$ solution and a cylindrical stainless steel rod of $25 \mathrm{~mm}$ diameter is put at the phantom center (Fig.- 5a). The stainless steel rod, called common mode electrode (CME) [17] in EIT, is connected to the ground point of the EIT electronics to reduce the common mode error [18] of the circuits.

\subsection{Data Collection}

$1 \mathrm{~mA}, 50 \mathrm{kHz}$ sinusoidal constant current is injected to the phantom boundary with and without inhomogeneity and the electrode potentials are collected using opposite current injection protocol (Fig.-5b). Nylon cylinders are put as the inhomogeneity near electrode no. 3, 5 and 7 and the current signal is injected through the electrode switching module. The boundary potentials developed at the surface electrodes placed on the phantom boundary are collected using opposite current injection protocol (Fig.-5b) and sent to the PC for the analysis. RMS potentials on all the electrodes are measured for first eight current projections and the voltage data set (contains 128 voltage data for a sixteen electrode system) are saved as a .txt file in PC for computation. Boundary data for first eight current projections are sufficient to produce impedance image (due to reciprocity principle) and that is why the other eight current projections are not required [16]. The boundary potentials $\left(\mathrm{V}_{1}\right.$ through $\mathrm{V}_{16}$ in Fig.$5 b)$ developed at the surface electrodes $\left(E_{1}, E_{2}, E_{3}, \ldots, E_{15}\right.$ and $\left.E_{16}\right)$ are collected for the first eight current projections. The potential profile of symmetric electrodes (SEP) [19] of the phantom is also studied for evaluating the boundary data profile of the EIT system. The axis through the current electrodes divides the electrodes (excluding the current electrodes) in two sets (all are symmetrically placed in space) which are called symmetric electrode pair [19]. The equipotential lines and the SEPs are shown in the Fig.-5b. All the electrodes in a SEP are symmetrically placed in space with respect to the axis through the current electrodes divides the electrodes (Fig.-5b). All the SEPs of a 16 electrode system for current projection 1 are shown in Fig.-5b and 6a.

\begin{tabular}{|c|c|c|}
\hline & First Seven measurements & Next Seven Measurements \\
\hline SEP1 & Electrode 2 & Electrode 16 \\
\hline SEP2 & Electrode 3 & Electrode 15 \\
\hline SEP3 & Electrode 4 & Electrode 14 \\
\hline SEP4 & Electrode 5 & Electrode 13 \\
\hline SEP5 & Electrode 6 & Electrode 12 \\
\hline SEP6 & Electrode 7 & Electrode 11 \\
\hline SEP7 & Electrode 8 & Electrode 10 \\
\hline SEP8 & Electrode 9 & Electrode 9 \\
\hline
\end{tabular}

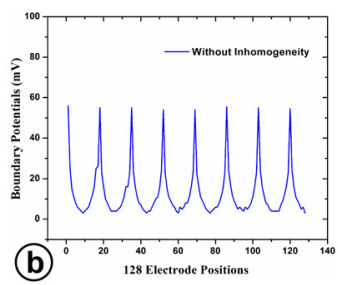

Fig. 6. (a) Symmetric electrode pairs (SEP) of a 16 electrode EIT system in current projection 1, (b) Boundary potentials for homogeneous medium.

\section{Results and Discussion}

The boundary potentials developed for homogeneous medium ( $\mathrm{NaCl}$ solution) show that (Fig.-6b) the surface potential profile changes in similar fashion as it changes for a wired EIT system in opposite method. It is observed that the potential profiles of all the current projections are symmetric (Fig.-6b) which indicates that the circular phantom system is symmetric and stabile. It is reported that [16] for opposite method, only first 128 voltages measured for first eight projections are independent data and hence are sufficient to reconstruct the inhomogeneities due to the reciprocity principle [16]. Hence the all the boundary potentials are collected for the first eight current projections and the 128 voltage data are studied (Fig.-6b). Consequently the acquisition time 
in this case reduces to $50 \%$ of the full acquisition time of the 16-electrode EIT system. The potential of the SEPs (Fig.-7a) also shows that the experimental phantom is symmetric with respect to the current injection axis. It is observed that the standard deviation of SEP1, SEP2, SEP3, and SEP4 are less than 1.0 whereas the standard deviation of SEP5, SEP6, SEP7, and SEP8 are zero (Fig.-7b).
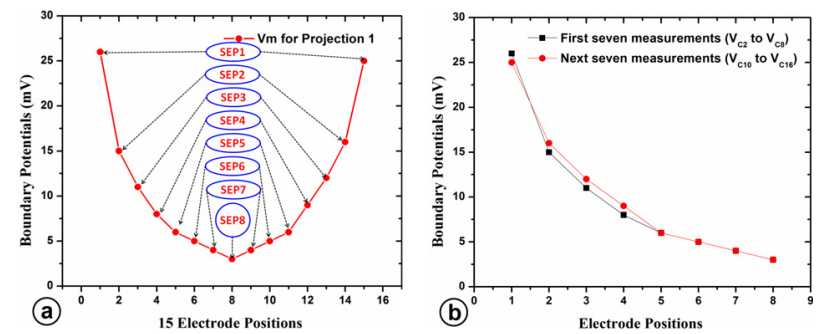

Fig. 7. (a) Boundary potentials in current projection 1 for the homogeneous medium, (b) SEP electrode potentials for first seven measurements and next seven measurements.

Circular nylon inhomogeneities are placed at the different electrode positions (Figs.-8a, 9a and 10a) and the RMS boundary potentials are measured using data acquisition system. Boundary data profiles (Figs. $-8 \mathrm{~b}, 9 \mathrm{~b}$ and $10 \mathrm{~b}$ ) of their corresponding phantom configurations (Figs.-8a, 9a and 10a respectively) show that the maximum potential $\left(\mathrm{V}_{\mathrm{ei}}\right)$ of the boundary data for a phantom with inhomogeneity placed near $\mathrm{N}^{\text {th }}$ electrode occurs in $[((\mathrm{N}-$ 1) $\times 16)+N]^{\text {th }}$ data point in the boundary potential data matrix.
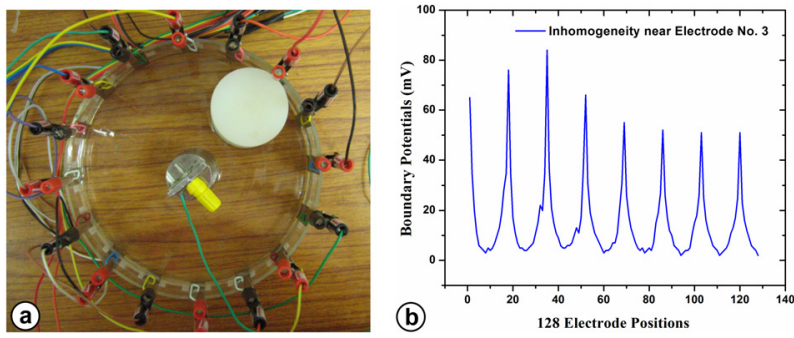

Fig. 8. (a) Practical phantom with nylon cylinder at electrode no. 3, (b) boundary potentials.

The maximum potential $\left(\mathrm{V}_{\mathrm{ei}}\right)$ point in the boundary potential data matrix is the potential of the electrode which is positioned near the inhomogeneity provided the positive terminal of the current source is connected to the same electrode. The voltage drop across the current path along the inhomogeneity and the electrode is found maximum because of the high resistance of inhomogeneity and the high electrode contact impedance. It is also observed that for all the inhomogeneity positions boundary data are successfully generated by the system. Boundary potentials measured with nylon inhomogeneity are found satisfactory for all the at electrode positions (Fig.-8a, 9a and 10a). Potential curves with nylon cylinder at electrode 3 (Fig.-8a) show that the high voltage peak is appeared at the $[((3-1) \times 16)+3]^{\text {th }}$ position or $35^{\text {th }}$ data point (Fig. $\left.-8 b\right)$ of the data matrix (in $3^{\text {rd }}$ projection loop). Hence it is understood that the inhomogeneity is positioned at the electrode number 3 . Potential curves with inhomogeneity at electrode 5 (Fig.-9a) also show that the high voltage peak is appeared at the $69^{\text {th }}$ data point (Fig. -9 b) of the data matrix (in $5^{\text {th }}$ projection loop) which clearly indicates the presence of inhomogeneity at $5^{\text {th }}$ electrode position. Similarly boundary data (Fig.-10b) for inhomogeneity at electrode 7 (Fig.-10a) show that the high voltage peak comes at the $103^{\text {th }}$ data point (Fig.-10b) of the data matrix (in $7^{\text {th }}$ projection loop). Hence it is understood that the inhomogeneity is positioned at the electrode number 3.
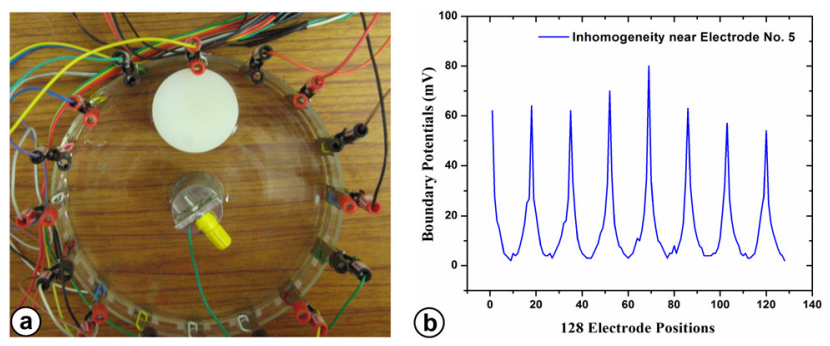

Fig. 9. (a) Practical phantom with nylon cylinder at electrode no. 5, (b) boundary potentials. 

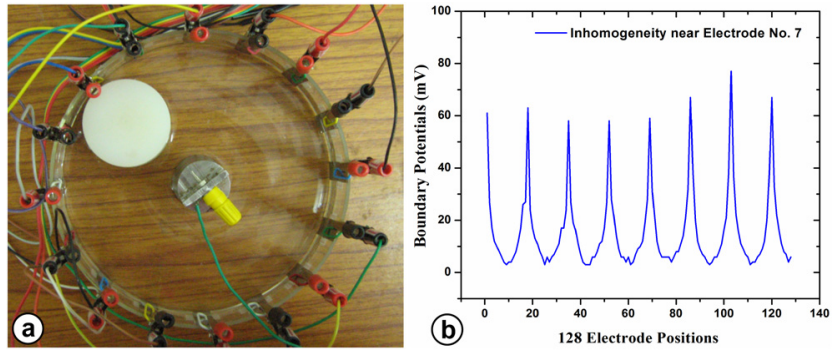

Fig. 10. (a) Practical phantom with nylon cylinder at electrode no. 7, (b) boundary potentials.

\section{Conclusions}

Surface electrode switching of 16-electrode wireless EIT is proposed and studied using a Radio Frequency (RF) based digital data transmission technique operating with 8 channel encoder/decoder ICs. Transmitter/receiver module developed is successfully interfaced with the USB based DAQ system through the analog multiplexers based electrode switching module. USB based data acquisition system properly generate digital data required for multiplexer operation. 8-bit parallel digital data are generated by DAQ card and sent to the transmission module which transmits the digital data bits to the receiver. Receiver module feeds the parallel digital bits to the binary adder circuits and the 16-bit data obtained at the adder outputs are fed to the multiplexers of the electrode switching module for surface electrode switching. Results show that the wireless module successfully transmits and receives the parallel data for switching the voltage and current electrodes wirelessly. $1 \mathrm{~mA}, 50 \mathrm{kHz}$ sinusoidal constant current is injected to the phantoms with different inhomogeneity configurations using opposite current injection protocol. The boundary potentials developed at the surface electrodes are measured and studied to assess the wireless data transmission. Boundary data collected from practical phantom show that the multiplexers are operating in the required sequence in opposite current protocol. The voltage picks obtained at the proper positions in the boundary data matrix proved that the sequential operation of multiplexers and successful wireless transmission of digital bits. The developed wireless digital data transmission module can be used for wireless EIT-data acquisition system.

\section{References}

[1] Webster J. G., Electrical impedance tomography. Adam Hilger Series of Biomedical Engineering, Adam Hilger, New York, USA 1990 .

[2] Metherall; Peter, Three Dimensional Electrical Impedance Tomography of the Human Thorax, PhD Thesis, University of Sheffield. January 1998.

[3] Denyer; C.W.L., Electronics for Real-Time and Three-Dimensional Electrical Impedance Tomographs, PhD Thesis, Oxford Brookes University, January 1996

[4] 4Bushberg; J. T., Seibert; J. A., Leidholdt Jr.; E. M., Boone; John M., The Essential Physics of Medical Imaging, 2nd Edition, Lippincott Williams \& Wilkins, ISBN-10: 0683301187.

[5] Li; Ying et al, A Novel Combination Method of Electrical Impedance Tomography Inverse Problem for Brain Imaging, IEEE Transactions on Magnetics, Vol. 41, No. 5, May 2005

[6] Bagshaw, Andrew P. et al; Electrical impedance tomography of human brain function using reconstruction algorithms based on the finite element method, NeuroImage 20 (2003) 752-764

[7] Murphy; D, Burton; P, Coombs; R, Tarassenko; L and Rolfe; P, Impedance Imaging in the Newborn, Clin. Phys. Physiol. Meas., 8, Suppl. A, pp 131-40, 1987.

[8] Hope; Tyna A. and Iles; Siân E, Technology review: The use of electrical impedance scanning in the detection of breast cancer, Breast Cancer Res 2004, 6:69-74

[9] Brown; B. H., "Medical impedance tomography and process impedance tomography: a brief review," Measurement Science \& Technology, vol. 12, pp. 991-996, Aug 2001.

[10] Linderholm; Pontus et al., Cell Culture Imaging Using Microimpedance Tomography, IEEE Transactions on Biomedical Engineering, Vol. 55, No. 1, January 2008

[11] Arpinar, V.E.; Eyuboglu, B.M.; Microcontroller controlled, multifrequency electrical impedance tomograph, Engineering in Medicine and Biology Society, 2001. Proceedings of the 23rd Annual International Conference of the IEEE

[12] Voldman S. H., ESD : RF Technology and Circuits, John Wiley \& Sons Ltd, The Atrium, Southern Gate, Chichester, 2006

[13] Bera T. K. and Nagaraju J., A Multifrequency Constant Current Source for Medical Electrical Impedance Tomography, Proceedings of the IEEE International Conference on Systems in Medicine and Biology 2010 (IEEE ICSMB 2010), 16 $6^{\text {th }}-18^{\text {th }}$ Dec'2010, Kharagpur, India, pp 278-283. DOI: 10.1109/ICSMB.2010.5735387

[14] Data Sheet, MAX038-High-Frequency Waveform Generator, Maxim Integrated Products, Inc. CA 94086. 
[15] Data Sheet, AD811 - High Performance Video Op Amp, Analog Devices, Inc.,

[16] Bera T. K. and Nagaraju J., Resistivity Imaging of A Reconfigurable Phantom With Circular Inhomogeneities in 2D-Electrical Impedance Tomography, Measurement, Volume 44, Issue 3, March 2011, Pages 518-526. DOI:10.1016/j.measurement.2010.11.015

[17] Bera T. K. and Nagaraju J., A Stainless Steel Electrode Phantom to Study the Forward Problem of Electrical Impedance Tomography (EIT), Sensors \& Transducers Journal, Vol. 104, Issue 5, May 2009, pp. 33-40

[18] Rosell J. and Riu P., Common-mode feedback in electrical impedance tomography, Clin. Phys. Physiol. Meas., 1992, Vol. 13, Suppl. 4 11-14.

[19] Bera T. K. and Nagaraju J., Studying the Boundary Data Profile of A Practical Phantom for Medical Electrical Impedance Tomography with Different Electrode Geometries, Proceedings of The World Congress on Medical Physics and Biomedical Engineering-2009 September 7 - 12, 2009, Munich, Germany, IFMBE Proceedings 25/II, pp. 925-929, 2009 